\title{
Mapping the functional brain state of a world champion freediver in static dry apnea
}

\author{
Jitka Annen ${ }^{1,2}$ - Rajanikant Panda ${ }^{1,2} \cdot$ Charlotte Martial $^{1,2} \cdot$ Andrea Piarulli $^{1,3} \cdot$ Guillaume Nery $^{4} \cdot$ \\ Leandro R. D. Sanz ${ }^{1,2}$. Juan M. Valdivia-Valdivia ${ }^{5,6}$. Didier Ledoux $x^{7,8} \cdot$ Olivia Gosseries $^{1,2} \cdot$ Steven Laureys $^{1,2}$
}

Received: 1 February 2021 / Accepted: 4 August 2021 / Published online: 22 August 2021

(c) The Author(s), under exclusive licence to Springer-Verlag GmbH Germany, part of Springer Nature 2021

\begin{abstract}
Voluntary apnea showcases extreme human adaptability in trained individuals like professional free divers. We evaluated the psychological and physiological adaptation and the functional cerebral changes using electroencephalography (EEG) and functional Magnetic Resonance Imaging (fMRI) to $6.5 \mathrm{~min}$ of dry static apnea performed by a world champion free diver. Compared to resting state at baseline, breath holding was characterized by increased EEG power and functional connectivity in the alpha band, along with decreased delta band connectivity. fMRI connectivity was increased within the default mode network (DMN) and visual areas but decreased in pre- and postcentral cortices. While these changes occurred in regions overlapping with cerebral signatures of several meditation practices, they also display some unique features that suggest an altered somatosensory integration. As suggested by self-reports, these findings could reflect the ability of elite free divers to create a state of sensory dissociation when performing prolonged apnea.
\end{abstract}

Keywords Voluntary apnea $\cdot$ Functional connectivity $\cdot$ EEG $\cdot$ MRI $\cdot$ Meditation $\cdot$ Mind-body dissociation

\section{Introduction}

Jitka Annen and Rajanikant Panda contributed equally as first authors.

Olivia Gosseries and Steven Laureys Contributed equally as last authors.

Jitka Annen

jitka.annen@uliege.be

1 Coma Science Group, GIGA Consciousness, University of Liège, Liège, Belgium

2 Centre du Cerveau, University Hospital of Liège, Liège, Belgium

3 Department of Surgical, Medical, Molecular and Critical Area Pathology, University of Pisa, Pisa, Italy

4 NERYBLUE, Nice, France

5 Department of Neurosurgery. St, Joseph's Hospital, Tampa, FL, USA

6 International Association for Development of Apnea (AIDA International), Medical and Science Committee, Zurich, Switzerland

7 Anesthesia and Intensive Care, GIGA Consciousness, ULiège, Liège, Belgium

8 Intensive Care Department, University Hospital of Liège, Liège, Belgium
As breathing is crucial to survival, the breathing reflex is difficult to override voluntarily for a sustained period of time. However, several professional (e.g., pearl divers) and sportive activities (e.g., underwater rugby) require periods of apnea. The central nervous system initiates and maintains respiration spontaneously via interconnected groups of neurons in the brainstem and the spinal cord (Mitchell and Berger 1975). These respiratory centers are located in the medulla oblongata (ventral respiratory group, dorsal respiratory group) and the pons (pontine respiratory group) which receive information from chemoreceptors sensitive to carbon dioxide $\left(\mathrm{CO}_{2}\right)$, oxygen $\left(\mathrm{O}_{2}\right)$ and $\mathrm{pH}$ levels, as well as different inputs from the central and peripheral nervous system. Among those, peripheral sensory feedback from the lungs and airways are responsible for several respiratory reflexes (e.g., sneeze, cough or Hering-Breuer reflexes). Similarly, top-down inputs from the cerebral cortex can override breathing control unconsciously (e.g., when speaking or singing) or consciously (e.g., in apnea (Lumb and Nunn 2010)). The brainstem respiratory centers integrate these regulating factors to generate the rate and pattern of breathing, and sends a neuronal drive to effector respiratory 
muscles (Horner and Bradley 2007) via descending pathways in the spinal cord. The resulting ventilation ensures energy production by the supply of $\mathrm{O}_{2}$ and the disposal of $\mathrm{CO}_{2}$.

A few studies investigated the effect of voluntary apnea (i.e. breath holding) on various visceral functions, including cardiovascular parameters (Javorka et al. 2002), hypercapnia (i.e., elevated $\mathrm{CO}_{2}$ blood levels) (Bain et al. 2017), oxygen saturation and muscle sympathetic nerve activity (Heusser et al. 2009). Lung packing is a breathing technique that pumps or pushes air into the lung, surpassing the total lung capacity (i.e., the maximum capacity of forced inhalation) by about 1-2 L. Lung packing specifically increases the amount of air available for ear equalization during depth diving, as well as the amount of air (and oxygen) in alveoli responsible for gas exchange (Schipke et al. 2015) prolonging the normoxic phase and delaying the onset of the hypoxic phase of apnea. Yet, during voluntary apnea, cerebral adaptations are observed, including increased arterial pressure, cerebral blood flow, and reduced cerebral metabolic rate of oxygen (see (Bain et al. 2018) for a review). However, early sensory processing and later cognitive processing seem unaffected in trained breath holders (Steinberg and Doppelmayr 2019). Furthermore, prolonged durations of apnea do not seem to induce long-term harmful effects on the brain of trained apneists (Overgaard et al. 2006).

The extreme human biological adaptability observed in trained individuals like free divers can lead to prolonged apnea for up to 24 min (with a preparatory phase of pure oxygen inhalation) in water benefitting from the diving response (i.e., the pause in respiration accompanied by reduced heart rate and increased in peripheral vascular resistance to promote blood flow to the brain and heart while restraining blood flow to non-essential muscles) (Foster and Sheel 2005). The contribution of psychological processes (e.g., mental control), together with physical ones (e.g., diving reflex) (Kawakami et al. 1967), seems crucial for the accomplishment of prolonged apnea (Panneton and Gan 2020). Yet, even without the contribution of the diving response, breath holding in dry conditions and without inhalation of pure oxygen is feasible up to about $7 \mathrm{~min}$, as shown in our experiment. Skin conductance and heart-related markers (e.g., heart rate) associated to stress, do not change during either the normoxic or hypoxic phase of breath holding, which suggests that elite apnea divers possess profound mental control (Laurino et al. 2012). This might mean that apneists do not respond directly to the alarming physiological signals, in favour of supporting the voluntary apnea task.

Trained free divers use an array of mental techniques to detach from the conscious mind (some called it the "monkey mind") and allow the subconscious mind to "take over the dive experience". Each practitioner is different, and there is variability in techniques and personal experience during a dive, yet the notion of positive feelings and happiness is universal. Some mental techniques to reach this state include mental training to detach from the "surface" or to think of a happy place, amongst many other techniques. Freedivingand specifically the depth discipline-is described by athletes as a moment of detachment from the senses, thought, fear, and/or time perception. The concept of time is fluid, with no tangible concept of "future" or "past", but only the "now". Mantras, for example, are commonly used during freediving, some including the phrases, among others: "let go", "trust", "only now exists", "I am here". Several other non-ordinary states of consciousness, such as meditation and hallucination experiences that are associated with similar feelings of well-being have been studied. They can be rather comparable to the reports of the subjective experience during free diving that are available online (e.g., an elite apneist who experiences "pure consciousness" 1 or the mental training practises for freedivers ${ }^{2}$ ). Moreover, emerging research based on traditional Indian practices suggests that breath holding might induce altered states of consciousness (Wasson 2013; Zaccaro and Penazzi 2019). In mice, the neural bases for the calming effect of controlled breathing as well as the balance between calm behaviour and stress have been attributed to a cell cluster in the medulla (Steriade 2006). In summary, the freediving experience can be described as peaceful, mystical, personal, and a means to look inside the self that might share characteristics with other states of altered consciousness such as during the use of psychedelic substances or Near-Death Experiences (NDE) (Martial et al. 2020).

Although there is evidence on both the physiological and psychological effects of breathing practices, their interdependence is poorly understood (Zaccaro et al. 2018). In the current study, we aim to investigate the neurobiology of the positive psychological effects of breathing control, and to test whether the cerebral changes observed during prolonged apnea can be attributed to the biological effects of prolonged voluntary apnea or also to the psychological state required to perform apnea. We hypothesize that if the cerebral functional changes are (only) related to altered biophysiological processes such as hypercapnia, the effects should build up slowly over time during the apnea period. Alternatively, if they represent a psychological change to achieve cognitive control aiming at overriding the breathing reflex and sustaining prolonged apnea, we suspect the alterations to be predominantly state-dependent. In the latter case, we furthermore hypothesize that the neural signature of prolonged voluntary apnea may be similar to what is observed in meditative states. This study will allow comparison with

\footnotetext{
${ }^{1}$ https://youtu.be/-MZetpFw7qY.

2 https://youtu.be/o3bB9sFXXHg.
} 
the existing literature on non-ordinary states of consciousness and may lead to the identification of a common neural substrate for these peculiar self-induced states. We present the case study of a 33-year-old elite free-diver world champion in constant weight apnea who performed prolonged periods of static dry apnea as well as resting state during high-density electroencephalogram (hd-EEG) and functional magnetic resonance imaging (fMRI) and provided a detailed report of his subjective experience.

\section{Materials and methods}

\section{Subject}

We investigated apnea-induced changes in brain function using hd-EEG and fMRI along with several measures of visceral functions in a 33-year-old right handed professional male free diver, former world champion in constant weight apnea ( $>30$ official competitions between 2001 and 2015). $\mathrm{He}$ started professional apnea training at the age of 15 . Since then, he has continuously practiced the discipline, and the maximum time without performing apnea was 1 month. He has been on an intense training schedule from 1996 till 2015, the year he retired from professional competitions (i.e., 1996-2000: 3-4 trainings per week, 2001-2015: 6-8 trainings per week, after 2015: 2-5 times per week). The experiment took place in 2016 . He performed meditation practices in 2011 for about 6-12 months, but not around the period of our study performed in 2016. His daily practice includes a brief yoga session (10-20 min) in the morning. Around the time of our study, he was also doing about 1 or 2 yoga sessions of 60-90 min every week. The apneist does not have any history of medical or psychological conditions.

\section{Apnea phenomenology}

Our elite diver subject provided a detailed verbal report of his practice and subjective experience during dry apnea in general, and specified if and how this was different from the conditions during the experiment. He also rated retrospectively both the descending and the ascending phase of a deep dive (thus not of the dry apnea for which the EEG and fMRI were acquired) using the Greyson Near-Death Experience (NDE) scale (Greyson 1983). The scale was thus used to characterize and quantify the apnea and post-apnea conditions at the phenomenological level, as performed in usual under water practice to assess the characteristics in ecological conditions. The self-reported scale includes 16 items assessing specific features, such as the experience of peacefulness, altered time perception, out-of-body experience and unusual sensations. Each item can be scored as 0 (i.e., "not present"), 1 (i.e., "mildly or ambiguously present") or 2 (i.e., "definitively present"). A total score (out of 32) is obtained by summing the scores of all items, reflecting the richness of the subjective experience. Total scores equal to or higher than 7 are considered to share features of a NDE.

The local ethics committee approved the study, and the subject provided written informed consent for study participation, in accordance with the declaration of Helsinki (1967) and its later amendments.

\section{Data acquisition}

Hd-EEG and MRI data were acquired on two consecutive days during eyes-closed awake resting state with normal breathing and during eyes-closed prolonged apnea. The subject was installed in supine position for both EEG and fMRI in a quiet, lighted room in the presence of the experimenters. Apnea here refers to the subject performing a complete dry, static and voluntary apnea with eyes closed for as long as the subject could sustain. The preparation for the apnea period started with buccal pumping (i.e., lung packing). When the subject was ready, the apnea started after a 10 -s countdown. During static dry apnea, the subject wore a nose clip. The tongue was not locked and the mouth was closed to not let air out. The glottis was closed but opened sometimes to build up some pressure for air to leave the mouth. Most of the time there was a bubble of air in the mouth as a habit taken from deep diving during which this is essential to keep equilibrium.

The apnea ended as soon as the subject felt the urge to breathe again, which was after $7 \mathrm{~min}$ for the EEG recording, and $6.5 \mathrm{~min}$ for the fMRI recording. During both experiments, an anesthesiologist monitored visceral functions, including the heart rate, oxygen saturation $\left(\mathrm{SpO}_{2}\right)$, end-tidal capnography $\left(\mathrm{EtCO}_{2}\right)$, and respiratory rate (based on nasal $\mathrm{CO}_{2}$ outflow) to ensure the subject's well-being and to measure the duration of the apnea period. The recorded data of the visceral functions during the fMRI experiment was not retrievable and only the signals during the EEG experiment could be analyzed (Fig. 1).

Hd-EEG was recorded with 256 electrodes (Hydrocel Geodesic Sensor Net with saline solution) at a sampling rate of $500 \mathrm{~Hz}$ (Electrical Geodesics Inc., Eugene, Oregon, USA). First, a baseline resting state recording of $10 \mathrm{~min}$ was acquired, followed by the apnea preparation and apnea recording of $7 \mathrm{~min}$.

Structural and functional MRI data were acquired using a 3-Tesla scanner (Siemens MAGNETOM Trio, Münich, Germany). Headphones were used to reduce the noise level and foam pads were used to increase comfort and stability on the scanner table. A 3D T1-weighted image was acquired with 120 slices $(\mathrm{TR}=2300 \mathrm{~ms}, \mathrm{TE}=2.47 \mathrm{~ms}$, flip angle $90^{\circ}$, voxel size $=1 \times 1 \times 1.2 \mathrm{~mm}^{3}, \mathrm{FOV}=256 \times 256 \mathrm{~mm}^{2}$ ). Resting state fMRI Bold Oxygen Level Dependent (BOLD) 
Fig. 1 Heart rate (blue line), pulse oximetry (red line) and end-tidal concentration of $\mathrm{CO}^{2}$ (black line) in the nasal respiratory flow during the apnea period, and one minute after the end of the apnea

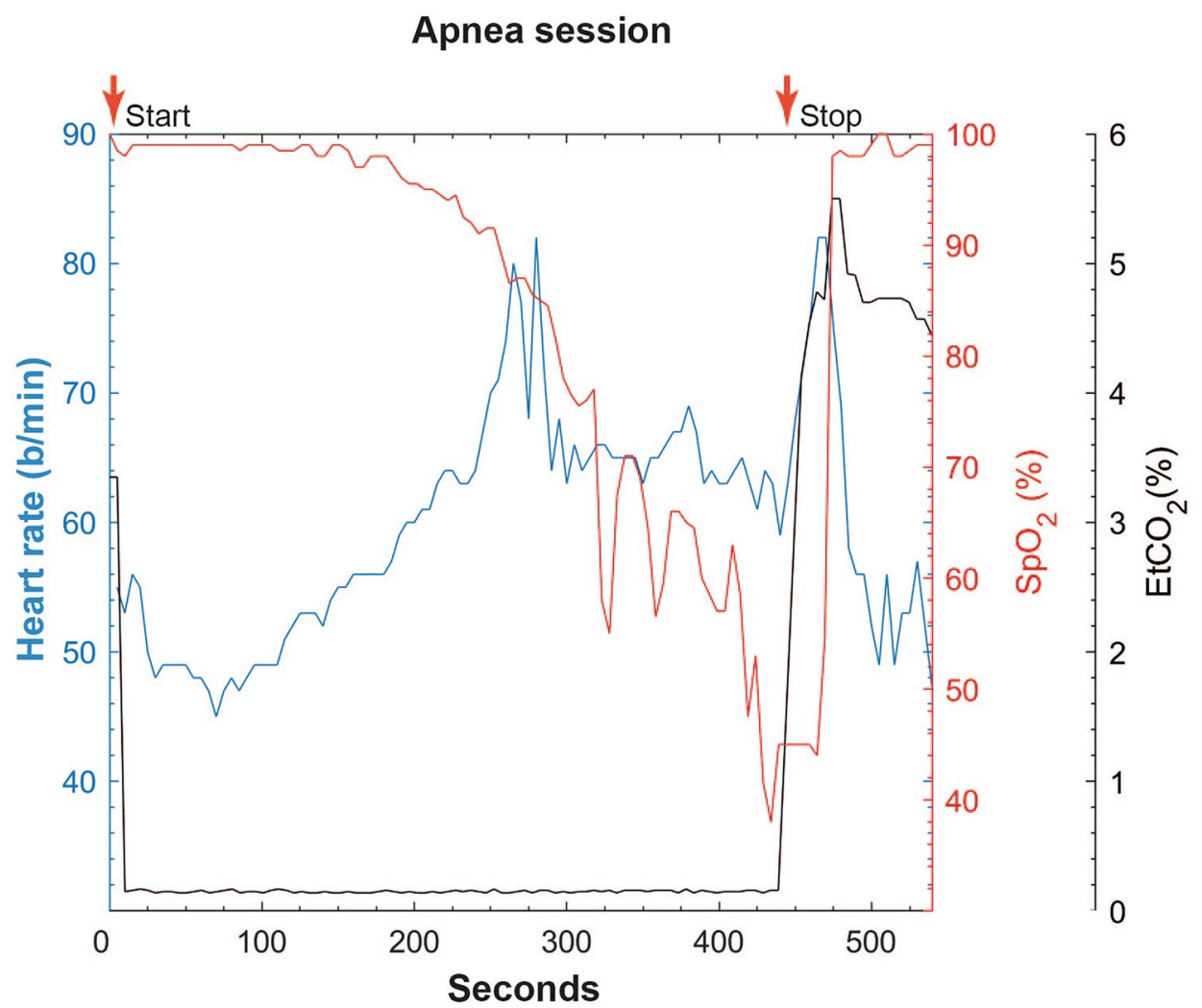

images were acquired using echo planar imaging sequence with the following parameters: 195 images, 32 slices, TR $2000 \mathrm{~ms}$, TE $30 \mathrm{~ms}$, FOV $192 \times 192 \mathrm{~mm}$, flip angle $78^{\circ}$, voxel size $3 \times 3 \times 3 \mathrm{~mm}$ ). Based on the duration of apnea during EEG, the resting state fMRI was recorded for $7 \mathrm{~min}$ prior to the apnea, and the apnea recording lasted $6.5 \mathrm{~min}$.

\section{(Electro)physiological data analysis}

Two-sample t-tests (two-tailed) were performed to assess mean differences in physiological signals across the time series. Measures were down-sampled to match the EEG epoch length of $10 \mathrm{~s}$. A linear regression was used to investigate whether the visceral signals (i.e., heart rate, $\mathrm{SpO} 2$ and $\mathrm{EtCO} 2$ ) showed a linear increase or decrease over time.

All the EEG analyses were performed using MATLAB. EEG data was down-sampled to $250 \mathrm{~Hz}$ and band-pass filtered between 0.7 and $45 \mathrm{~Hz}$ using EEGLAB toolbox (version 13.5.1 (Delorme and Makeig 2004)). Facial and neck electrodes $(n=83)$ were removed resulting in a dataset consisting of 173 electrodes. Bad channels and bad epochs were discarded by visual inspection. From the EEG data of the apnea condition, the first $10 \mathrm{~s}$ and the last $50 \mathrm{~s}$ of the data were discarded due to noise, and the remaining 6 min data were analyzed. For consistency, the first $10 \mathrm{~s}$ of the resting state data were discarded and the following 6 min of clean EEG data was used for analysis. Independent Component
Analysis (ICA) using the 'runica' algorithm implemented in EEGLAB was carried out to remove ocular, muscular and ECG artifacts from the data. Bad channels were interpolated using spline interpolation, and the data were re-referenced to the average reference.

The comparison between baseline and apnea was conducted using a sliding window approach with 10-s epochs without overlap between continuous windows, totaling 36 epochs. Spectral power in the delta $(1-4 \mathrm{~Hz})$, theta $(4.1-8 \mathrm{~Hz})$, alpha $(8.1-13 \mathrm{~Hz})$, beta $(13.1-20 \mathrm{~Hz})$ and gamma (20-45 Hz, (Steriade 2006)) bands were estimated for bins of $0.5 \mathrm{~Hz}$ between 0.5 and $30 \mathrm{~Hz}$, for each epoch using a multitaper method(Percival and Walden 1993). Subsequently, mean channel power density was estimated averaging between epochs. For each region of interest (ROI: midline, right and left frontal, parietal, central and temporal, please refer to Fig. 3 for a map with electrodes selected for the different ROIs), the mean power density was then estimated by averaging among channels included in the ROI and the resulting value was log-transformed. Connectivity matrices were calculated for each epoch and each frequency band. Connectivity was estimated using weighted phase lag index (wPLI) (Hardmeier et al. 2014) between each pair of electrodes using 'wPLI function' implemented in the fieldtrip toolbox (Vinck et al. 2011). Regional connectivity was calculated for the 12 ROIs per epoch, frequency band and condition. The connectivity between two ROIs ' $\mathrm{i}$ ' and ' $\mathrm{j}$ ' 
was estimated by averaging the wPLI indexes of all possible pairs of electrodes ' $x$ ' and ' $y$ ' such that $x \in i$ and $y \in j$ (Teipel et al. 2018). The whole brain connectivity over the epochs was represented in the 3D arcs head view (King et al. 2013).

Condition-mediated differences between ROI band power and connectivity were assessed using two-sample (twotailed) $t$-tests, False Discovery Rate (FDR) corrected (Yoav Benjamini and Yosef Hochberg 1995) for the 12 ROIs. A linear regression was used to investigate whether the connectivity values changed linearly over time, suggesting that prolonged apnea-duration would aggravate cerebral changes. Finally, a linear regression was employed to investigate whether the significant EEG connectivity changes depended on visceral parameters (i.e., heart rate, $\mathrm{SpO} 2$ and EtCO2). Results were considered significant at FDR corrected $p<0.05$.

\section{fMRI analysis}

fMRI data were preprocessed using slice time correction and by realignment of all volumes to the first volume. The 3D T1-weighted MRI was segmented and normalized to MNI152 standard space of $2 \times 2 \times 2 \mathrm{~mm}^{3}$ using Statistical Parametric Mapping 12 (https://www.fil.ion.ucl.ac.uk/ $\mathrm{spm} / \mathrm{software} / \mathrm{spm} 12 /$ ). The head motion parameters were computed for the transverse and rotation axis. There was no difference in the head motion between the baseline [translation $=(0.09,0.70,0.76 \mathrm{~mm})$; rotation $=\left(0.015^{0}, 0.006^{0}\right.$, $\left.0.006^{0}\right)$ ] and the apnea state [translation $=(0.55,0.56$, $0.88 \mathrm{~mm})$; rotation $\left.=\left(0.020^{0}, 0.006^{0}, 0.012^{0}\right)\right]$. Before computing the connectivity maps, the head motion and physiological source noise correction was carried out using the CompCor algorithm as implemented in CONN toolbox (http://www.nitrc.org/projects/conn). Then, the data were band-pass filtered between 0.01 and $0.1 \mathrm{~Hz}$. The first 6 min of fMRI data were analyzed for the baseline state and apnea conditions.

A sliding window approach, similar to the EEG analysis, was applied with 12 segments of $30 \mathrm{~s}$. Voxel-wise wholebrain global functional connectivity (GFC) was performed using the CONN-functional connectivity toolbox. The GFC is a measure of global coherence, which computes the average connectivity of every seed voxel to the entire brain [15, 16]. The Fisher's z-transformations of GFC maps were computed for both conditions. Differences in GFC between baseline and apnea were estimated with the contrast $[-1$, 1]. Results were considered significant after FDR-correction (i.e., whole-brain voxel-wise at $p<0.05$ ), and minimum cluster extent threshold of 20 contiguous voxels (Bharath et al. 2017).

The average of z-transformed GFC values (effect size) were extracted for every $30 \mathrm{~s}$ window for apnea and baseline conditions. $T$-tests (two-tailed) were performed to evaluate differences in state-dependent (increased and decreased) connectivity. A linear regression was used to investigate if the effect size values per state (i.e., increased and decreased connectivity) showed a trend over time.

\section{Results}

\section{Phenomenological report of the apnea experience}

In general, during the resting state before the breath hold the subject focusses on breathing in a calm manner comparable to a meditative or hibernation state. The ultimate way for him to relax is to focus on the duration of inspiration, breath hold and expiration. The duration of the expiration phase is at least double of the inspiration and breath hold, after which the subject expires through a small hole between the teeth and tongue, making a hissing sound. The subject is very much focused on time, and wants to fully experience breathing. During apnea itself, the subject does not stop thinking, but everything seems to slow down. The breath hold period, in general and during the experiment, can be divided in four distinct phases. First, the transition period to the apnea "state of mind" which is limited to the first minute of apnea. Then, from minute 1 till about $4.5 \mathrm{~min}$ into the apnea, the apneist feels very good. Happiness takes over, and he feels to be in the "here and now" without focusing on time. He does not need anything, to breathe or move, and experiences moments of "pure consciousness". However, most of the time there is an ongoing analysis of the pleasant feelings and thoughts he is experiencing. He tries to extend this period of good feelings for the longest time possible, until transitioning in the third phase of the apnea. The first contractions of the chest signal the body's urge to breathe, and the initial discomfort fades away leaving the subject feeling good again even if these chest contractions continue to occur involuntarily. This is the moment when the apneist sets his goal on the apnea duration, as he knows the apnea has lasted about $5 \mathrm{~min}$, and starts the last 1.5 min countdown. During this fourth phase of the apnea, he tries to get a taste of every second passing by, accepts it, and does not rush thoughts or anything else. He focusses on maintaining his rhythm to achieve the goal he set. This last phase is characterized by feelings of real euphoria (i.e., the good feelings and happiness are even more intense) and potential loss of lucidity, yet, thanks to his excellent training, our subject keeps control over the situation. The urge to breathe in this last phase is less profound than during the third phase of the apnea period.

When recalling the descending phase of the deep dive in water the apneist scores 9 out of 32 on the Greyson NDE scale (Greyson 1983) while for the ascending phase of the deep dive he scores 14 out of 32 . Both phases could be 
considered a NDE-like phenomenology, as they are above the cut-off score of $7 / 32$.

In our experimental setting, especially in the MRI, the subject reported to feel some anxiety and pressure to perform. However, as soon as the apnea started, the anxiety was replaced by a complete concentration on the exercise and his experience was similar as the usual practice described above. At the end of the two breath holds in experimental environment setting, the subject felt happiness to have accomplished his goals in the particular scientific setting. Before performing a deep dive, our apneist usually performs a visualization exercise, but not before the static dry apnea in this experiment.

\section{Physiological signals}

Heart rate (normal values about $60-100$ beats per minute) was higher during apnea $(M=59, \mathrm{SD}=8.7)$ than during resting state $[M=50, \mathrm{SD}=1.7 ; t(37.9)=-6.3$, $p<0.001,95 \%$ CI $[-12.2,-6.2)]$. EtCO2 (normal values about 4.6-5.9 $\mathrm{kPa})$ was lower during apnea $(M=0.15 \mathrm{kPa}$, $\mathrm{SD}=0.007)$ than during resting state $(M=4.6 \mathrm{kPa}, \mathrm{SD}=0.6$; $t(35.0)=47.8, p<0.001,95 \% \mathrm{CI}(4.2,4.6)] . \mathrm{SpO} 2$ (normal values about $95-100 \%)$ was lower during apnea $(M=90.0$, $\mathrm{SD}=12.6)$ than during resting state $(M=98.2, \mathrm{SD}=1.0$; $t(35.4)=3.9, p<0.001,95 \% \mathrm{CI}(4.0,12.5)]$.

During apnea, we observed no linear change in $\mathrm{EtCO} 2$, while increasing heart rate $\left(R^{2}=0.72, F(1,34)=86.6\right.$, $p<0.001 ; \beta=4.06, p<0.001)$ and decreasing $\mathrm{SpO} 2$ $\left(R^{2}=0.72, F(1,34)=88.1, p<0.001 ; \beta=-5.92, p<0.001\right)$ were measured over time (Fig. 1).

\section{EEG signals}

During apnea, an increase in delta power was observed over the right frontal, temporal and parietal, and left central and temporal cortices ( $p<0.05$ for all comparisons, Fig. 2). Furthermore, alpha power was increased over the right central cortex, the left temporal cortex and bilaterally over the occipital cortex ( $p<0.05$ for all comparisons, Fig. 2).

Within delta band, whole-brain connectivity during apnea $(M=0.025, \mathrm{SD}=0.01)$ was globally decreased as compared to baseline $(M=0.035, \mathrm{SD}=0.01)(t(50.7)=4.14$, $p<0.001,95 \%$ CI $(0.005,0.015)$; Fig. 3a). On the contrary, alpha band whole-brain connectivity was globally increased during apnea $(M=0.045, \mathrm{SD}=0.025)$ compared to baseline $(M=0.024, \mathrm{SD}=0.01)(t(45.12)=-4.75, p<0.001,95 \%$ CI $(-0.03,-0.01)$; Fig. 3b). Looking at regional differences, we observed decreased delta connectivity between left frontal and left occipital, between left central and left occipital, between left temporal and right parietal, and between right parietal and lower midline areas. In contrast, we found increased alpha connectivity between bilateral frontal, parietal, central, temporal and occipital regions, predominantly in the right hemisphere, and along the midline (Fig. 3c, d). We did not find connectivity differences either in theta, beta or gamma frequency bands.

During baseline, delta band connectivity values fluctuated, but did not follow a linear trend over time $\left(R^{2}=0.006\right.$, $F(1,34)=0.2, p=0.65)$. In contrast, during apnea, delta band connectivity $(\beta=0.001)$ slightly increased with time $\left(R^{2}=0.13, \mathrm{~F}(1,34)=5.3, p=0.028\right)$. However, the two connectivity time series did not show any significant betweencondition difference $(p=0.18)$. When considering the alpha band, there was no evidence for a trend in connectivity over time either for baseline $\left(R^{2}=0.01, \mathrm{~F}(1,34)=0.37, p=0.55\right)$ or apnea $\left(R^{2}=0.01, F(1,34)=0.5, p=0.49\right)$. Analogously to delta band, no between-condition difference was observed $(p=0.67)$.

During the resting state baseline condition, none of the physiological measures were related to the observed connectivity either in delta $\left(R^{2}=0.05, F(3,32)=0.61, p=0.62\right)$ or alpha $\left(R^{2}=0.02, F(3,32)=0.21, p=0.89\right)$ bands. During the apnea period, none of the physiological measures were related to the observed connectivity in delta $\left(R^{2}=0.08, F(3\right.$, $32)=0.98, p=0.41)$ either. However, when considering the alpha band, we found evidence of a moderate contribution of SpO2 ( $\beta=0.001, p=0.047)$ on functional connectivity, although the total model of the physiological data did not significantly explain the alpha connectivity $\left(R^{2}=0.13, F(3\right.$, $32)=1.63, p=0.2)$.

\section{fMRI BOLD signals}

We observed brain regions with increased and other brain regions with decreased GFC during apnea as compared to baseline (Fig. 4). For increased GFC, the effect size of baseline $(M=0.72, \mathrm{SD}=0.13)$ was higher than that of apnea $(M=-0.94, \mathrm{SD}=0.13 ; t(22)=-31.6, p<0.001,95 \% \mathrm{CI}$ $(-1.77,-1.55)]$. Similarly, for decreased GFC, the effect size of baseline was also higher $(M=1.18, \mathrm{SD}=0.17)$ than that of apnea $(M=-0.54, \mathrm{SD}=0.16 ; t(22)=25.6$, $p<0.001,95 \%$ CI $(1.58,1.86)]$. During apnea, increased GFC was found in a number of subcortical regions such as bilateral thalamus, caudate, and right amygdala. Increased GFC was also measured in cortical territories, including regions pertaining to the default mode network (DMN) and to the executive control network (ECN): precuneus, frontal (bilateral middle frontal, superior frontal cortex, frontal pole, and right inferior frontal), angular and left occipital pole cortices. In parallel, decreased connectivity was apparent mainly in the sensory-motor area (bilateral precentral, postcentral, superior parietal lobule and anterior cingulate), right supramarginal, left superior temporal gyrus, right 

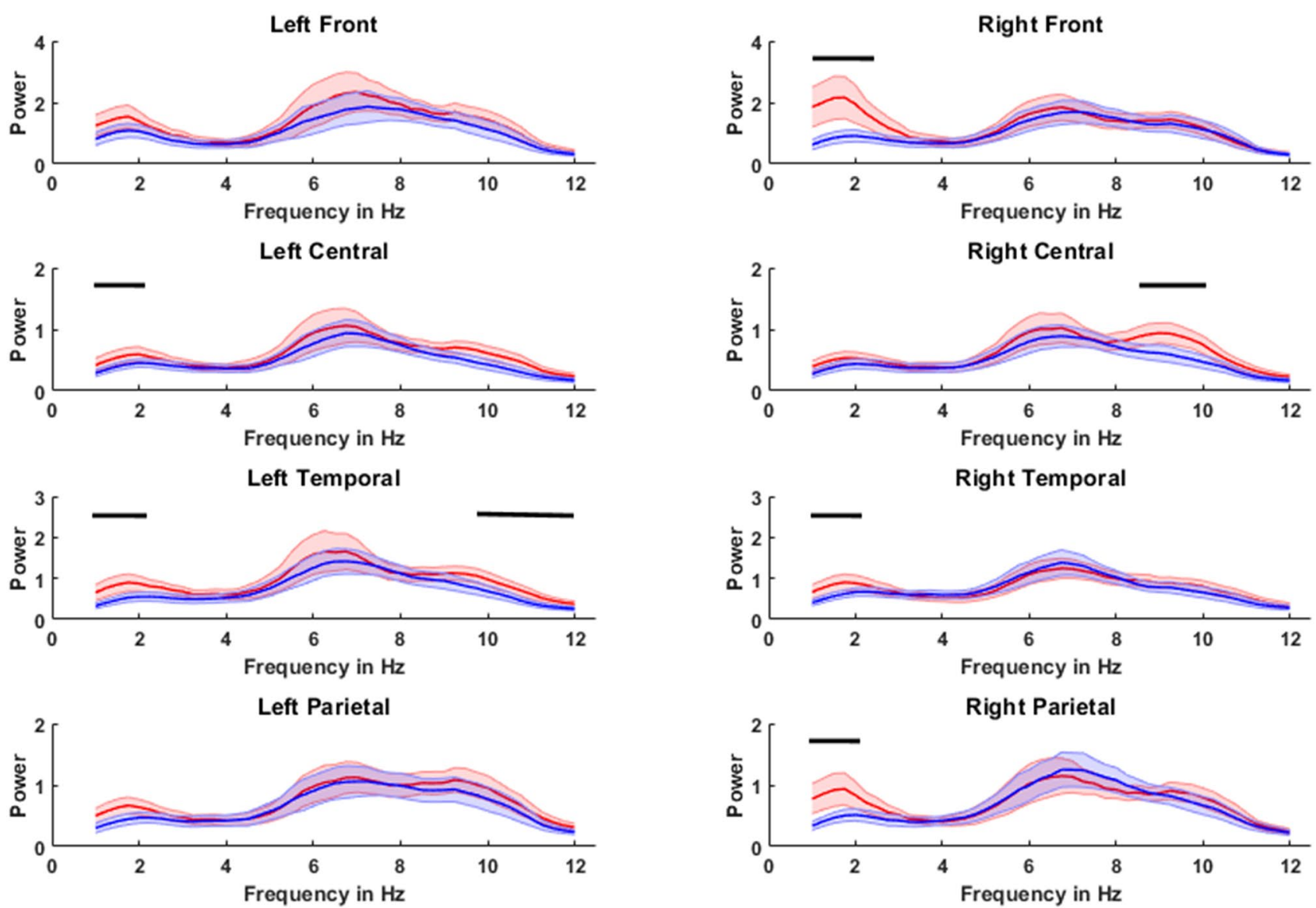

Left Occipital
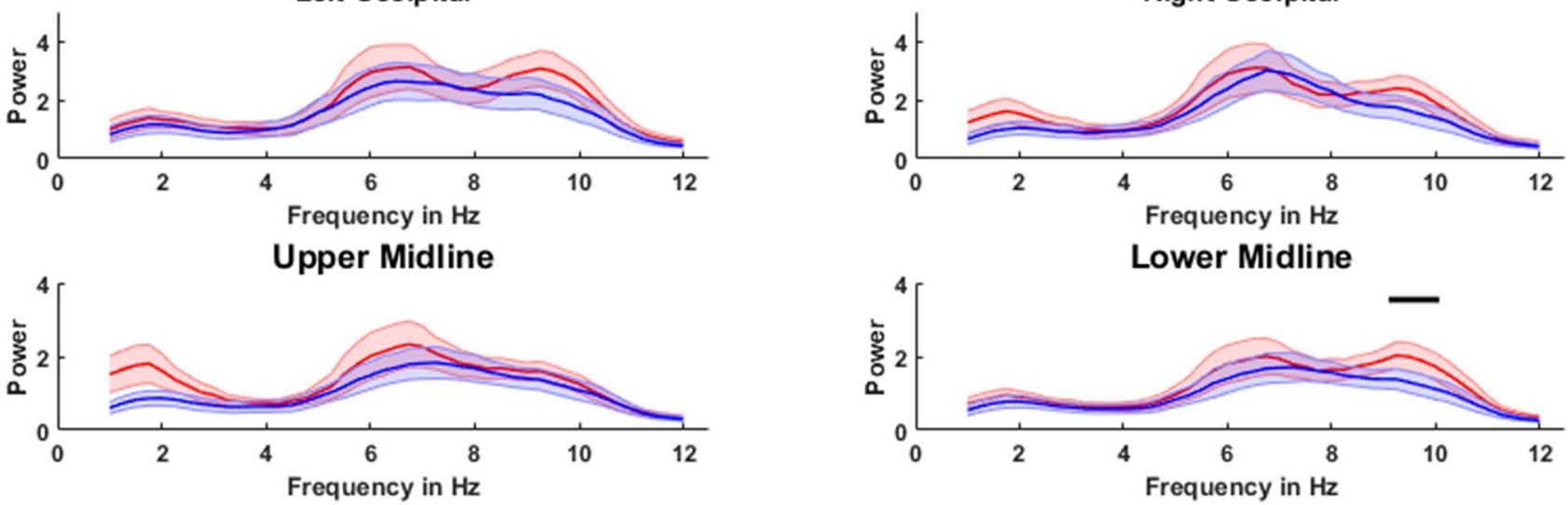

Fig. 2 Power spectral density of baseline (blue line) and apnea (red line) states in the delta, theta and alpha frequency range. Lines above the graph represent significant differences in power between baseline and apnea at $p<0.05$ (FDR corrected)

insula, bilateral lingual gyrus and left occipital fusiform gyrus (Fig. 4, Supplementary Table 1).

The effect size for brain connectivity was not modulated by time either for positive (baseline: $R^{2}=0.03, F$ $(1,10)=0.3, p=0.61$; apnea: $R^{2}=0.17, F(1,10)=2.1$, $p=0.18$ ) or negative (baseline: $R^{2}=0.02, F(1,10)=0.3$, $p=0.62$; apnea: $\left.R^{2}=0.08, F(1,10)=0.8, p=0.38\right)$ connectivity, and the slopes did not differ for the two conditions (all $p>0.05$ ). 


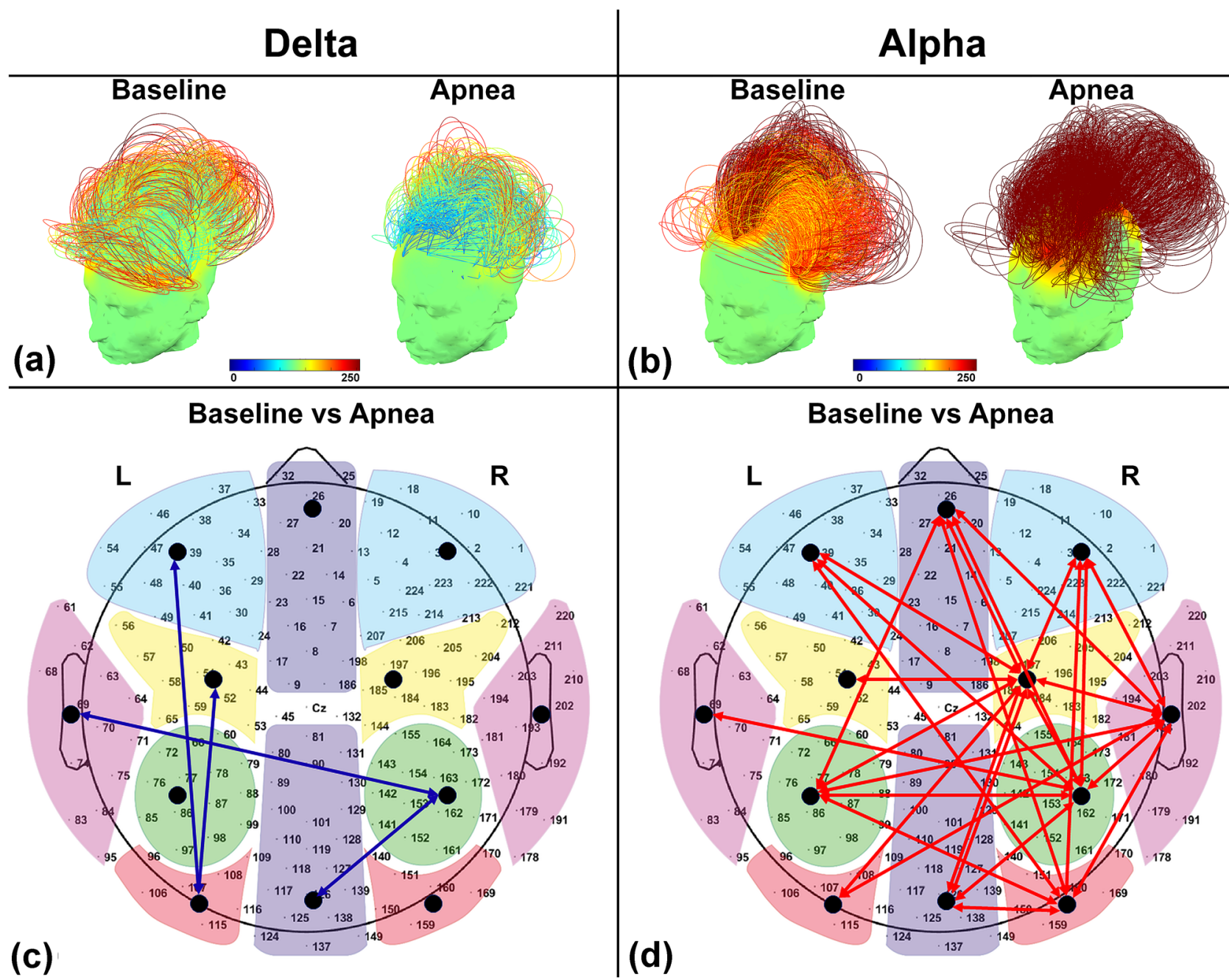

Fig. 3 EEG-based connectivity in the delta $\mathbf{a}$ and alpha $\mathbf{b}$ frequency bands, during baseline and apnea conditions. The arcs' height and color represent connectivity strength (assessed with weighted phase lag index), where low arcs and blue/yellow colors represent weak connectivity and high arcs and orange/red colors represent strong connectivity. The scalp color represents the number of connection (i.e., degree) of each node (i.e., electrode). Significant differences in regional connectivity between the baseline versus apnea in the delta

band $\mathbf{c}$ and alpha band $\mathbf{d}$ were observed ( $p<0.05$, FDR corrected). Blue lines represent decreased connectivity, which were observed in the delta band over the left hemisphere and posterior interhemispheric connections. The red lines represent increased connectivity during apnea as compared to baseline, observed mainly observed between regions within the right hemisphere as well as for interhemispheric connections

\section{Discussion}

The elite apnea diver presents a breath-holding induced mental state that is characterized by psychological, physiological and cerebral alterations. We obtained three important findings investigating functional brain connectivity during voluntary apnea. We observed an instantaneous cerebral signature of prolonged apnea that does not show increased adaptation over time. This signature comprises increased EEG connectivity in alpha band and a concurrent decrease in delta band as compared to rest.

Finally, during apnea, fMRI connectivity analyses showed increased global connectivity in subcortical (thalamus and amygdala) and cortical regions (precuneus, frontal and occipital cortices), paralleled by decreased connectivity over sensorimotor, temporal and lingual areas.

We propose that the observed cerebral changes reflect a mental state characterized by a feeling of well-being, or even "pure consciousness" (Metzinger 2020) alternating with (or alongside) an increase in cognitive control required to sustain a considerable period of voluntary apnea and required to stop the apnea at the most critical but also most pleasant phase. Indeed, this hypothesis is supported by the detailed 


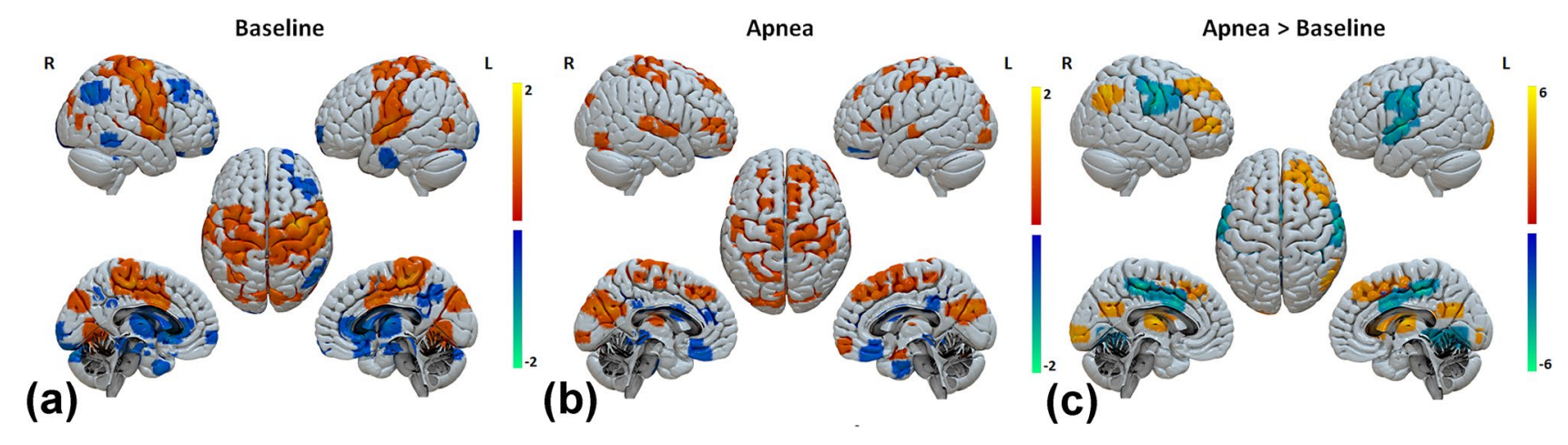

Fig. 4 Voxel-wise whole-brain global functional connectivity (GFC) of baseline $\mathbf{a}$ and apnea $\mathbf{b}$ states presented on the MNI template brain. During baseline, a relative strong (in red) functional connectivity of the somatosensory cortex and occipital cortex and a weak (in blue) functional connectivity of the subcortical areas and lateral frontoparietal cortex can be observed. During apnea, strong functional connectivity can be observed widespread throughout the cortex and in the thalamus, while the functional connectivity is low in the frontal pole. The time series show the stable effect sizes for the regions with connectivity and for weak connectivity over time. Differences between

personal report given by the world champion regarding his training practice and his subjective experience during the experiments. Elite divers emphasize the psychological rather than the physical difficulties they endure during their activities. They report that meditation and yoga exercises are important for their preparation, and that relaxation during the apnea period is beneficial to reduce oxygen consumption. Several meditation practices are associated with characteristics beneficial for breathing control. For example, transcendental meditators perform more shallow inspiration whilst at the same time showing a reduced response to the effects of hypercapnia (Wolkove et al. 1984). Likewise, transient decreased cerebral oxygen level has been suggested to explain the emergence of NDE-like experiences (Martial et al., 2020), with which the deep-dive apnea experience shares features. Thus, meditation and breathing control seem to occur in symbiosis and might promote altered states of consciousness. Although several breath holding techniques are associated with positive feelings and pleasant sensations (Zaccaro et al. 2018), voluntary apnea might be a distinct state that requires additional cognitive control to endure the pain in the third phase of the apnea, as well as to start breathing again during the euphoric fourth phase.

The heart rate of our subject during apnea increased from his rather low resting state heart rate, possibly compensating a decreased cardiac output (Schipke et al. 2015). Blood $\mathrm{O}_{2}$ saturation levels were lower during the period of apnea as compared to resting state, but the average $\mathrm{SpO}_{2}$ did not drop below 90\%, a threshold under which it is considered harmful for patients with sleep apnea (Nieto et al. 2000). The harmless nature of voluntary apnea is supported by the functional connectivity of the resting state and apnea conditions c considered significant at $p<0.05$, voxel-wise whole-brain correction (FDR corrected). Red color represents increased connectivity and blue color represents decreased connectivity during apnea as compared to baseline. A relative increased functional connectivity is present in the thalamus, caudate, amygdala, frontal, occipital and posterior cingulate cortices during apnea as compared to baseline. Relative decreased functional connectivity during apnea can be observed in the pre- and postcentral, anterior cingulate and temporo-parietal cortex as well as the insula

the absence of neurocognitive decline in a group of elite apnea divers (Ridgway and McFarland 2006). As the $\mathrm{EtCO}_{2}$ measure employed in our study depends on nasal respiration, absent during the apnea period, it does not reflect the blood $\mathrm{CO}_{2}$ levels during the apnea period and no conclusions can be drawn regarding the presence of hypercapnia (i.e., increased $\mathrm{CO}_{2}$ levels). Apnea induced functional cerebral changes could be attributed to gradual alterations in the cerebral metabolic rate of oxygen, driven by hypoxia (i.e., decreased $\mathrm{O} 2$ levels) or more likely hypercapnia (Bain et al. 2017). If all cerebral changes could be attributed to this process, the build-up of $\mathrm{CO}_{2}$ over time would be accompanied by increasing cerebral effects during the apnea. However, in our elite free diver, we did not observe a continuous inor decrease of spectral changes but rather an instantaneous change in the cerebral signature (both for EEG and fMRI) of apnea. For this reason, we believe our results do not solely reflect the effect of hypercapnia or a global change in blood oxygenation. Previous studies are in line with our findings showing that apnea-induced physiological changes in the alpha band are probably not related to hypercapnia (Schellart and Reits 1999). Furthermore, a group study showed that 5 min of apnea does not cause notable hypoxia or impaired cerebral performance/attention among trained apnea performers (Ratmanova et al. 2016). Also fMRI functional connectivity is unlikely to be largely affected by hypercapnia, as we would expect to see an hypercapnia-induced increase in the functional connectivity of all resting state networks (Hou et al. 2019). Thus, it seems that the observed brain changes cannot be fully attributed to the effects of hypercapnia, and the potential interplay between the psychological 
effects and the observed changes in cerebral function merit further exploration.

The neurophysiological signature of apnea as measured with EEG shows important overlaps with several cerebral characteristics of cognitive control and meditation practices. Just like during meditation practices that require to shift focus, we found an increase in delta power during apnea, highlighting a potential role of delta power in attentional engagement (Lee et al. 2018). This is in line with the direct increase of delta power after apnea initiation in non-trained breath holders (Morelli et al. 2015). The increased delta power during apnea could be related to inhibition of sensory processing, in favor of internal concentration and reducing activity of unnecessary networks to accomplish a task (Harmony 2013). Related to that, the reduced connectivity in the delta band is in accordance with what has been found in several meditation techniques, which is speculated to be related to altered subjective experience of detachment, and letting go (Lehmann et al. 2012). In a yoga master, increased alpha power has been reported during meditation (Arambula et al. 2001), much like with the apneist in the current study, although decreased alpha power has also been reported during voluntary apnea (Steinberg et al. 2017). Increased alpha power has been found while focusing on breathing (Braboszcz and Delorme 2011) as well in several meditative practices (see (Cahn and Polich 2013) for review). Likewise, increased alpha phase coherence has been associated to transcendental meditation performance and is suggested to be related to improved mind-body health (Hebert et al. 2005). Indeed, increased alpha power has been associated to cognitive control (Sadaghiani and Kleinschmidt 2016), which is also required to successfully complete the breath holding task. Although scalp-level EEG cannot be used to make inferences about sources, the DMN could potentially be related to the observed effects in the alpha band (Chen et al. 2008).

The fMRI results show important spatial overlap with the neural correlates of voluntary breathing (i.e. isocapnic hyperpnea) in healthy volunteers who display increased neural activity in the primary sensory and motor cortices, supplementary motor area, cerebellum, thalamus, caudate nucleus, globus pallidum and medulla (McKay et al. 2003). During voluntary apnea the breath-holder showed an involvement of the same cortical areas, yet connectivity in motor areas and the cerebellum decreased. Furthermore, our fMRI findings show a striking similarity with observations during mindfulness (Tang et al. 2015) and Vipassana meditation (Chiesa 2010), including alterations of thalamo-cortical dynamics. Specifically, the thalamus, caudate, amygdala, precuneus, medial and lateral frontal and lateral occipital cortex show increased functional connectivity during apnea. These regions do not only belong to the DMN, but also to the executive control network (ECN) (Heine et al. 2012).
Increased medial PFC connectivity during voluntary apnea has been associated with the decision procedure, awareness, mentalizing abilities, potentially related to the decision to stop or prolong the apnea period (Akani et al. 2021). The increased functional connectivity of both networks might hint to an adaptation of the attentional system that enables fluctuations between the DMN and the ECN, potentially mediated by the locus coeruleus (Melnychuk et al. 2018). Indeed, in pranayama meditation, DMN activity and breathing (reflected by $\mathrm{CO}^{2}$ levels) are directly related (Melnychuk et al. 2018). Increased thalamic and caudate connectivity show resemblance with the cerebral dynamics observed during higher-order mental processes (Min 2010; Ward 2013; Saalmann 2014).

Contrasting with meditative states, we found increased functional connectivity of the precuneus during apnea. The precuneus is an important nucleus of the DMN (Fransson and Marrelec 2008; Utevsky et al. 2014), and could potentially be involved in balancing the altered cerebral sensoryrelated dynamics with the thalamus and frontal areas (Cunningham et al. 2017). Increased activation of the precuneus area might also decrease the attention towards irrelevant stimuli ( $\mathrm{Ng}$ et al. 2007), which in the case of sustained apnea could help to ignore the physiological urge to breathe during the third phase of the apnea. There is more fMRI-based evidence that points towards the dissociation between mind and body during apnea: the anterior cingulate, secondary motor, lingual, and superior temporal-parietal cortex as well as the insula showed decreased functional connectivity. The reduced insular activity is in contrast with findings observed during mindfulness meditation, where the insula is hypothesized to be recruited for self-regulation of attention, emotion and awareness (Tang et al. 2015). As the activation of the insula is directly related to subjective bodily and emotional feelings (Craig 2011), the reduced connectivity during apnea might contribute to the dissociation between the body and the brain. Indeed pain perception during the chest convulsions might be modulated by the insula (Wiech et al. 2010). The insular cortex has also been identified as locus for mediating autonomic nervous system responses (Cechetto 2014). Furthermore, there is more activation of the bilateral motor cortex at baseline and upon static apnea the motor cortices reduce their activity, consistent with a detachment from movement. Indeed, activity in the pre- and postcentral somatosensory areas is involved in the somatotopic representation of the body in space (Reed 2002), and might reflect respiratory sensations as observed in a respiratory-related evoked potential (Von Leupoldt et al. 2010). Our fMRI (and EEG) results suggest that the right hemisphere is more dominantly affected by apnea than the left hemisphere. It seems that the right cerebral hemisphere leads the perception of the physical and emotional self (Devinsky 2000). Furthermore, altered DMN activity might be also related 
to an altered sense-of-self (Qin and Northoff 2011), potentially related to a stronger sense of introspection. Moreover, when compared to baseline, the bilateral calcarine areas show increased activation during static apnea (fMRI) and the occipital areas displayed increased alpha power (EEG), which would reflect visualization techniques used during competitive breath-holding, although this is less important for the preparation of static apnea for our subject. The alterations caused by voluntary apnea as measured with fMRI are immediate and stable over time, supporting the notion of state change rather than dynamical change. The ensemble of these regions altered by apnea in our participant might thus be associated with a mind-body control leading to impaired integration of somatosensory input and altering the processing of bodily self-consciousness in the apnea state.

Other examples of individuals with extraordinary psychological skill set associated with specific cerebral function exist. The "Iceman" performs a forced breathing technique combined with meditation to endure cold-induced pain better and to experience euphoria, possibly through the release of endogenous opioids/cannabinoids (Muzik et al. 2018). Matthieu Ricard, Buddhist monk, can willfully induce meditative mental states that reduces the response to TMS stimulation in the "thoughtless" meditative state, even more than during drowsiness (Bodart et al. 2018). An expert in performing cognitive trance, a state of suppressed external awareness and increased internal awareness induced using traditional Mongolian practice, is able to induce measurable decreases and increases of cortical response to stimulation in regions associated to external and internal awareness (Gosseries et al. 2020). Likewise, the current study provides preliminary evidence that the voluntary apnea as performed by our subject increases the sense of well-being, alongside an increased autonomic control. While acknowledging that our study presents only one highly trained individual, we believe that such case reports have scientific value as preliminary evidence, and may eventually lead to the identification of a common neural substrate for these peculiar self-induced states. All these "athletes of the mind" use different techniques with different psychological and cerebral effects, yet are all associated with increased well-being and might eventually provide insights for alternative, drug-free therapies for psychological issues (e.g., post-traumatic stress disease or chronic pain) or pathologies of the autonomous nervous system.

The results of the presented study should be interpreted with caution due to several factors. First, we present a case study which limits the generalizability of our results. However, our record-holder and world champion participant is unique and therefore a group study would be challenging to perform. Furthermore, it was logistically impossible to obtain repeated measurements of the same subject performing apnea. In order to carefully interpret the results, we used stringent statistics to ensure the validity of our results. Second, due to the non-invasive character of the study we have only access to $\mathrm{EtCO}_{2}$ capnography. This solely measures the end-tidal breath concentration of $\mathrm{CO}_{2}$, but not circulating blood $\mathrm{CO}_{2}$ levels. We are therefore unable to quantify accurately the potential contribution of hypercapnia to the observed physiological changes. Moreover, our results are obtained in dry conditions and hence cannot be directly translated to the effects of apnea during water immersion with a diving response. Given the importance of the brainstem and in particular of the medulla, it would have been interesting to investigate the implications of those regions with fMRI. However, the brainstem (excluding the medulla) has only recently been added in the AAL3 atlas (Rolls et al. 2020). Last, even if the fMRI and EEG experiments were performed under equal conditions, the sequential nature of the acquisitions hampers the direct comparison of the fMRI and EEG results.

In conclusion, we show evidence for the presence of strong and widespread effects of apnea in a single subject. The ensemble of cerebral adaptations provides, for the first time, an indication that psychological effects are involved to the cerebral adaptations induced by or necessary to perform apnea. We pose that a meditation-like process acting as psychological facilitator, alongside an important dissociation between the body physiology and the mind, are instrumental for sustaining prolonged voluntary dry, static apnea. The cognitive control associated with voluntary apnea might increase the quality of life in clinical populations who would benefit from reduced salience of the autonomous nervous system, such as chronic pain. Further studies are required to replicate our findings and test these hypotheses regarding the apnea-induced altered state of consciousness in a larger sample and potentially more natural conditions.

Supplementary Information The online version contains supplementary material available at https://doi.org/10.1007/s00429-021-02361-1.

Acknowledgements The authors thank the whole staff from the Radiodiagnostic department of the University Hospital of Liège for the help in data acquisition and Benedetta Cecconi for the helpful discussion.

Author's contributions JA, RP, AP, SL and OG designed research. JA, $\mathrm{AP}, \mathrm{CM}, \mathrm{GN}$, DL and SL acquired the data. JA, RP and AP analysed the data. JA, RP, CM, AP, LRDS, JMV-V, SL and OG interpreted the results. SL and OG supervised research. JA drafted the manuscript. All authors contributed to result interpretation and the editing of the manuscript.

Funding The study was supported by the University and University Hospital of Liège, the Belgian National Funds for Scientific Research (FRS-FNRS), the European Union's Horizon 2020 Framework Programme for Research and Innovation under the Specific Grant Agreement No. 945539 (Human Brain Project SGA3), the European Space Agency (ESA) and the Belgian Federal Science Policy Office 
(BELSPO) in the framework of the PRODEX Programme, the Bial Foundation, the Mind Science Foundation, the fund Generet of the King Baudouin Foundation, the Mind-Care foundation and AstraZeneca Foundation. RP and LS are research fellows, OG is research associate, and SL is research director at FRS-FNRS.

\section{Declarations}

Conflict of interest The authors declare no conflict of interest.

Ethical approval The local ethics committee approved the study.

Consent to participate The subject provided written informed consent for study participation, in accordance with the declaration of Helsinki (1967) and its later amendments.

Consent for publication The authors affirm that human research participants provided informed consent for publication of the images in Figure(s) 1, 2, 3, 4. The participant provided informed consent regarding publishing their data and submission of the case report to the journal.

Data availability The data that support the findings of this study are available from the senior author (Olivia Gosseries (ogosseries@uliege. be) and Steven Laureys (Steven.Laureys@uliege.be) from University of Liege) upon reasonable request.

Code availability The EEGLAB toolbox code used for EEG preprocessing analyses is freely available online (https://sccn.ucsd.edu/ eeglab/download.php). The Fieldtrip toolbox code used for EEG frequency analyses and EEG connectivity analyses is freely available online (https://www.fieldtriptoolbox.org/download/). The CONN toolbox code used for fMRI connectivity analyses is freely available online (http://www.nitrc.org/projects/conn). The "Surf Ice" code used for fMRI connectivity results to display in a glass brain is freely available online (https://www.nitrc.org/projects/surfice/).

\section{References}

Akani A, Karidioula H, Bony K et al (2021) Feasibility of detecting brain areas involved in extreme breath-hold diving. J Neurol Res Ther 3:1-8. https://doi.org/10.14302/issn.2470

Arambula P, Peper E, Kawakami M, Hughes Gibney K (2001) The physiological correlates of Kundalini Yoga meditation: a study of a yoga master. Appl Psychophysiol Biofeedback 26:147-153. https://doi.org/10.1023/a:1011343307783

Bain AR, Ainslie PN, Barak OF et al (2017) Hypercapnia is essential to reduce the cerebral oxidative metabolism during extreme apnea in humans. J Cereb Blood Flow MeTab 37:3231-3242. https://doi. org/10.1177/0271678X16686093

Bain AR, Drvis I, Dujic Z et al (2018) Physiology of static breath holding in elite apneists. Exp Physiol 103:635-651. https://doi. org/10.1113/EP086269

Bharath RD, Panda R, Saini J et al (2017) Dynamic local connectivity uncovers altered brain synchrony during propofol sedation. Sci Rep 7:1-10. https://doi.org/10.1038/s41598-017-08135-2

Bodart O, Fecchio M, Massimini M et al (2018). SC Brain Stimul. https://doi.org/10.1016/j.brs.2018.08.018

Braboszcz C, Delorme A (2011) Lost in thoughts: neural markers of low alertness during mind wandering. Neuroimage 54:3040-3047. https://doi.org/10.1016/j.neuroimage.2010.10.008
Cahn BR, Polich J (2013) Meditation states and traits: EEG, ERP, and neuroimaging studies. Psychol Conscious Theory Res Pract 1:48-96. https://doi.org/10.1037/2326-5523.1.s.48

Cechetto DF (2014) Cortical control of the autonomic nervous system. Exp Physiol 99:326-331. https://doi.org/10.1113/expphysiol. 2013.075192

Chen ACN, Feng W, Zhao H et al (2008) EEG default mode network in the human brain: spectral regional field powers. Neuroimage 41:561-574. https://doi.org/10.1016/j.neuroimage.2007.12.064

Chiesa A (2010) Vipassana meditation: systematic review of current evidence. J Altern Complement Med 16:37-46. https://doi.org/ 10.1089/acm.2009.0362

Craig ADB (2011) Significance of the insula for the evolution of human awareness of feelings from the body. Ann N Y Acad Sci 1225:7282. https://doi.org/10.1111/j.1749-6632.2011.05990.x

Cunningham SI, Tomasi D, Volkow ND (2017) Structural and functional connectivity of the precuneus and thalamus to the default mode network. Hum Brain Mapp 38:938-956. https://doi.org/10. 1002/hbm.23429

Delorme A, Makeig S (2004) EEGLAB: an open source toolbox for analysis of single-trial EEG dynamics including independent component analysis. J Neurosci Methods 134:9-21. https://doi.org/10. 1016/j.jneumeth.2003.10.009

Devinsky O (2000) Right cerebral hemisphere dominance for a sense of corporeal and emotional self. Epilepsy Behav 1:60-73. https:// doi.org/10.1006/ebeh.2000.0025

Foster GE, Sheel AW (2005) The human diving response, its function, and its control. Scand J Med Sci Sport 15:3-12. https://doi.org/ 10.1111/j.1600-0838.2005.00440.x

Fransson P, Marrelec G (2008) The precuneus/posterior cingulate cortex plays a pivotal role in the default mode network: evidence from a partial correlation network analysis. Neuroimage 42:1178-1184. https://doi.org/10.1016/j.neuroimage.2008.05.059

Franzmeier N, Caballero MÁA, Taylor ANW et al (2017) Resting-state global functional connectivity as a biomarker of cognitive reserve in mild cognitive impairment. Brain Imaging Behav 11:368-382. https://doi.org/10.1007/s11682-016-9599-1

Gosseries O, Fecchio M, Wolff A et al (2020) Clinical neurophysiology. Clin Neurophysiol 131:586-588. https://doi.org/10.1016/j. clinph.2019.11.011

Greyson B (1983) The near-death experience scale. J Nerv Ment Dis 171:369-375

Hardmeier M, Hatz F, Bousleiman H et al (2014) Reproducibility of functional connectivity and graph measures based on the phase lag index (PLI) and weighted phase lag index (wPLI) derived from high resolution EEG. PLoS One 9:e0108648. https://doi.org/10. 1371/journal.pone. 0108648

Harmony T (2013) The functional significance of delta oscillations in cognitive processing. Front Integr Neurosci 7:1-10. https://doi. org/10.3389/fnint.2013.00083

Hebert R, Lehmann D, Tan G et al (2005) Enhanced EEG alpha timedomain phase synchrony during transcendental meditation: implications for cortical integration theory. Signal Process 85:22132232. https://doi.org/10.1016/j.sigpro.2005.07.009

Heine L, Soddu A, Gómez F et al (2012) Resting state networks and consciousness: alterations of multiple resting state network connectivity in physiological, pharmacological, and pathological consciousness states. Front Psychol 3:1-12. https://doi.org/10. 3389/fpsyg.2012.00295

Heusser K, Dzamonja G, Tank J et al (2009) Cardiovascular regulation during apnea in elite divers. Hypertension 53:719-724. https://doi. org/10.1161/HYPERTENSIONAHA.108.127530

Horner RL, Bradley TD (2007) Update in sleep and control of ventilation 2006. Am J Respir Crit Care Med 175:426-431. https://doi. org/10.1164/rccm.200701-043UP 
Hou X, Liu P, Gu H et al (2019) Estimation of brain functional connectivity from hypercapnia BOLD MRI data: validation in a lifespan cohort of 170 subjects. Neuroimage 186:455-463. https://doi.org/ 10.1016/j.neuroimage.2018.11.028

Javorka M, Žila I, Javorka K, Čalkovská A (2002) Do the oscillations of cardiovascular parameters persist during voluntary apnea in humans? Physiol Res 51:227-238

Kawakami Y, Natelson BH, DuBois AR (1967) Cardiovascular effects of face immersion and factors affecting diving reflex in man. $\mathrm{J}$ Appl Physiol 23:964-970. https://doi.org/10.1152/jappl.1967. 23.6.964

King JR, Sitt JD, Faugeras F et al (2013) Information sharing in the brain indexes consciousness in noncommunicative patients. Curr Biol 23:1914-1919. https://doi.org/10.1016/j.cub.2013.07.075

Lal C, Strange C, Bachman D (2012) Neurocognitive impairment in obstructive sleep apnea. Chest 141:1601-1610. https://doi.org/ 10.1378/chest.11-2214

Laurino M, Menicucci D, Mastorci F et al (2012) Mind-body relationships in elite apnea divers during breath holding: a study of autonomic responses to acute hypoxemia. Front Neuroeng 5:1-10. https://doi.org/10.3389/fneng.2012.00004

Lee DJ, Kulubya E, Goldin P et al (2018) Review of the neural oscillations underlying meditation. Front Neurosci 12:1-7. https://doi. org/10.3389/fnins.2018.00178

Lehmann D, Faber PL, Tei S et al (2012) Reduced functional connectivity between cortical sources in five meditation traditions detected with lagged coherence using EEG tomography. Neuroimage 60:1574-1586. https://doi.org/10.1016/j.neuroimage.2012. 01.042

Lumb AB, Nunn JF (2010) Nunn's applied respiratory physiology. Chap. 4, Churchill Livingstone, Elsevier, Edinburgh

Martial C, Cassol H, Laureys S, Gosseries O (2020) Near-death experience as a probe to explore (disconnected) consciousness. Trends Cogn Sci 24:173-183. https://doi.org/10.1016/j.tics.2019.12.010

McKay LC, Evans KC, Frackowiak RSJ, Corfield DR (2003) Neural correlates of voluntary breathing in humans. J Appl Physiol 95:1170-1178. https://doi.org/10.1152/japplphysiol.00641.2002

Melnychuk MC, Dockree PM, O’Connell RG et al (2018) Coupling of respiration and attention via the locus coeruleus: effects of meditation and pranayama. Psychophysiology 55:13091. https://doi.org/ 10.1111/psyp.13091

Metzinger T (2020) Minimal phenomenal experience meditation, tonic alertness, and the phenomenology of "pure" consciousness. Philos Mind Sci 1:1-44

Min BK (2010) A thalamic reticular networking model of consciousness. Theor Biol Med Model 7:1-18. https://doi.org/10.1186/ 1742-4682-7-10

Mitchell RA, Berger AJ (1975) Neural regulation of respiration. Am Rev Respir Dis 111:206-224. https://doi.org/10.1007/ 978-3-642-40308-8_2

Morelli MS, Vanello N, Giannoni A et al (2015) Correlational analysis of electroencephalographic and end-tidal carbon dioxide signals during breath-hold exercise. Proc Annu Int Conf IEEE Eng Med Biol Soc EMBS 2015-Nov:6102-6105. https://doi.org/10.1109/ EMBC.2015.7319784

Muzik O, Reilly KT, Diwadkar VA (2018) "Brain over body"—a study on the willful regulation of autonomic function during cold exposure. Neuroimage 172:632-641. https://doi.org/10.1016/j.neuro image.2018.01.067

Ng CW, Noblejas MI, Rodefer JS et al (2007) Double dissociation of attentional resources: prefrontal versus cingulate cortices. J Neurosci 27:12123-12131. https://doi.org/10.1523/JNEUROSCI. 2745-07.2007

Nieto FJ, Young TB, Lind BK et al (2000) Association of sleepdisordered breathing, sleep apnea, and hypertension in a large community-based study. Sleep Heart Health Study. JAMA 283(14):1829-1836. https://doi.org/10.1001/jama.283.14.1829

Overgaard K, Friis S, Pedersen RB, Lykkeboe G (2006) Influence of lung volume, glossopharyngeal inhalation and PET O2 and PET CO2 on apnea performance in trained breath-hold divers. Eur J Appl Physiol 97:158-164. https://doi.org/10.1007/ s00421-006-0156-2

Panneton WM, Gan Q (2020) The mammalian diving response: inroads to its neural control. Front Neurosci 14:1-27. https:// doi.org/10.3389/fnins.2020.00524

Percival DB, Walden AT (1993) Spectral analysis for physical applications: Multitaper and conventional univariatetechniques. Cambridge University Press, Cambridge

Qin P, Northoff G (2011) How is our self related to midline regions and the default-mode network? Neuroimage 57:1221-1233. https://doi.org/10.1016/j.neuroimage.2011.05.028

Ratmanova P, Semenyuk R, Popov D et al (2016) Prolonged dry apnoea: effects on brain activity and physiological functions in breath-hold divers and non-divers. Eur J Appl Physiol 116:1367-1377. https://doi.org/10.1007/s00421-016-3390-2

Reed CL (2002) Tactile perception. In: Ramachandran VS (ed) Encyclopedia of the Human Brain. Academic Press, San Diego, CA, pp 545-556

Ridgway L, McFarland K (2006) Apnea diving: long-term neurocognitive sequelae of repeated hypoxemia. Clin Neuropsychol 20:160-176. https://doi.org/10.1080/13854040590947407

Rolls ET, Huang CC, Lin CP et al (2020) Automated anatomical labelling atlas 3. Neuroimage 206:116189. https://doi.org/10. 1016/j.neuroimage.2019.116189

Saalmann YB (2014) Intralaminar and medial thalamic influence on cortical synchrony, information transmission and cognition. Front Syst Neurosci 8:1-8. https://doi.org/10.3389/fnsys.2014. 00083

Sadaghiani S, Kleinschmidt A (2016) Brain networks and $\alpha$-oscillations: structural and functional foundations of cognitive control. Trends Cogn Sci 20:805-817. https://doi.org/10.1016/j. tics.2016.09.004

Schellart NAM, Reits D (1999) Voluntary breath holding affects spontaneous brain activity measured by magnetoencephalography. Undersea Hyperb Med 26:229-234

Schipke JD, Kelm M, Siegmund K et al (2015) "Lung packing" in breath hold-diving: an impressive case of pulmo-cardiac interaction. Respir Med Case Rep 16:120-121. https://doi.org/10.1016/j. rmcr.2015.09.007

Steinberg F, Doppelmayr M (2019) Neurocognitive markers during prolonged breath-holding in freedivers: an event-related EEG study. Front Physiol 10:1-13. https://doi.org/10.3389/fphys.2019. 00069

Steinberg F, Pixa NH, Doppelmayr M (2017) Electroencephalographic alpha activity modulations induced by breath-holding in apnoea divers and non-divers. Physiol Behav 179:90-98. https://doi.org/ 10.1016/j.physbeh.2017.05.028

Steriade M (2006) Grouping of brain rhythms in corticothalamic systems. Neuroscience 137:1087-1106. https://doi.org/10.1016/j. neuroscience.2005.10.029

Tang YY, Hölzel BK, Posner MI (2015) The neuroscience of mindfulness meditation. Nat Rev Neurosci 16:213-225. https://doi.org/ $10.1038 / \mathrm{nrn} 3916$

Teipel S, Bakardjian H, Gonzalez-Escamilla G et al (2018) No association of cortical amyloid load and EEG connectivity in older people with subjective memory complaints. NeuroImage Clin 17:435-443. https://doi.org/10.1016/j.nicl.2017.10.031

Utevsky AV, Smith DV, Huettel SA (2014) Precuneus is a functional core of the default-mode network. J Neurosci 34:932-940. https:// doi.org/10.1523/JNEUROSCI.4227-13.2014 
Vinck M, Oostenveld R, Van Wingerden M et al (2011) An improved index of phase-synchronization for electrophysiological data in the presence of volume-conduction, noise and sample-size bias. Neuroimage 55:1548-1565. https://doi.org/10.1016/j.neuroimage. 2011.01.055

Von Leupoldt A, Vovk A, Bradley MM et al (2010) The impact of emotion on respiratory-related evoked potentials. Psychophysiology 47:579-586. https://doi.org/10.1111/j.1469-8986.2009.00956.x

Ward LM (2013) The thalamus: gateway to the mind. Wiley Interdiscip Rev Cogn Sci 4:609-622. https://doi.org/10.1002/wcs.1256

Wasson D (2013) Pause in breath: potential for altered states of consciousness in traditional Indian performance. In: Embodied Conscious Perform Technol 91-101. https://doi.org/10.1057/97811 37320056

Wiech K, Lin CS, Brodersen KH et al (2010) Anterior insula integrates information about salience into perceptual decisions about pain. $\mathrm{J}$ Neurosci 30:16324-16331. https://doi.org/10.1523/JNEUROSCI. 2087-10.2010

Wolkove N, Kreisman H, Darragh D et al (1984) Effect of transcendental meditation on breathing and respiratory control. J Appl
Physiol Respir Environ Exerc Physiol 56:607-612. https://doi.org/ 10.1152/jappl.1984.56.3.607

Benjamini Y, Hochberg Y (1995) Controlling the false discovery rate: a practical and powerful approach to multiple testing. J R Stat Soc Ser B 57:289-300

Zaccaro A, Penazzi G (2019) Neurophysiological model of altered states of consciousness induced by breathing techniques. Cosm Hist 15:210-224

Zaccaro A, Piarulli A, Laurino M et al (2018) How breath-control can change your life: a systematic review on psycho-physiological correlates of slow breathing. Front Hum Neurosci 12:1-16. https:// doi.org/10.3389/fnhum.2018.00353

Publisher's Note Springer Nature remains neutral with regard to jurisdictional claims in published maps and institutional affiliations. 